INFLUENCE OF THE SOLID INNER CORE AND COMPRESSIBILITY OF THE FLUID CORE ON THE EARTH NUTATION

\title{
Sergel DIAKONOV
}

\section{INTRODUCTION}

While calculating low frequency oscillations of the Earth liquid core spherical harmonic representation of the deformation field is usually used [1-3]:

$\mathbf{u}=\sum \sum\left[\mathrm{s}_{\mathrm{l}}^{\mathrm{m}}+\mathrm{T}_{\mathrm{l}}^{\mathrm{m}}\right]$.

Substitution of (1) into the equations of motion gives an infinite system $_{m}$ of differential equations for scalar functions $S_{l}{ }^{m}$ and $T_{L}{ }^{m}$. Approximate solutions of such a system are obtained by truncating of the system. But results of [4] show that sometimes such method divergences.

2 NUTATION OF THE EARTH WITH COMPRESSIBLE CORE

2. 1 MODEL. Let us calculate a forced nutation amplitude for the Earth model consisting of the rigid mantle and compressible liquid core with simple liquid density distribution:

$\rho\left(r_{\theta}\right)=\rho_{0}\left(1-\delta r_{\theta}^{2}\right)$,

where $r_{0}$ is dimensionless radius.

Let us investigate behavior of such a system affected by tide-generating potential. The mantle angular velocity may be written in the form:

$\Omega=\{\eta(1-i j) \operatorname{ext}(i \sigma t)+k\} \omega$,

and nutation amplitude $\eta$ must be found.

2.2 EQUATIONS OF LIQUID OSCILLATIONS. Small oscillations of the fluid core are described by the following equations:

$\Delta \psi-\frac{4 \omega^{2}}{\sigma^{2}} \frac{\partial^{2} \psi}{\partial z^{2}}=$

$=-\left(\sigma^{2}-4 \omega^{2}\right) \frac{\psi-\left[V_{1}+V_{t}+\eta \omega^{2}(x z-i y z) \exp (i \sigma t)\right]}{\alpha^{2}}-\frac{\sigma^{2}-4 \omega^{2}}{i \sigma} \vee \frac{\nabla_{\rho}}{\rho}$,

$\Delta \mathrm{V}_{1}=4 \pi \mathrm{G} \rho\left(1 / \alpha^{2}\right)\left(\psi-\mathrm{V}_{1}-\mathrm{V}_{\mathrm{t}}-\eta \omega^{2}(x z-i y z) \exp (i \sigma t)\right)$

$\psi=P_{1} / \rho+V_{1}+V_{t}+\eta \omega^{2}(x z-i y z) \exp (i \sigma t)$,

Here $v, P_{1}, V_{1}$ are perturbations of liquid velocity, pressure and gravitansional potential correspondingly, $a$ is speed of sound and $V_{t}$ is tide-generating potential. 
2. 3 METHOD OF SOLUTION. Let us represent the solution in the form:

$\psi=\psi_{\mathbf{P}}+\delta \psi$,

where $\psi_{p}$ is the Poincare solution:

$\psi_{\mathbf{P}}=-$ now $\frac{1-1 / k-\tau^{2} /\left(1-\varepsilon_{c}^{2}\right)}{1-1 / k+\tau^{2} /\left(1-\varepsilon_{c}^{2}\right)}(x z-i, z z), \quad k=\sigma / 2 \omega, \tau^{2}=1-1 / k^{2}$.

Let represent $\delta \psi$ by expansion on characteristic functions of the Poincare operator:

$\delta \psi=\sum_{m}\left\{\sum_{k}\left\{a_{L k}^{m} \Psi_{l k}^{m}\right\} \exp (i o t)\right.$.

and $\Psi_{l k}^{m}$ functions satisfy to the equation

$\left(\Delta-\frac{4 \omega^{2}}{\sigma^{2}} \frac{\partial^{2}}{\partial_{z}^{2}}\right) \Psi_{l k}^{m}=\lambda_{l k}^{m} \Psi_{l k}^{m}$

Substitution of (7) and (9) into the system (4)-(5) gives a system of equations for $a_{l k}$. Nondiagonal elements of the matrix of this system, as numerical results show, are small compared to the diagonal ones. This allows the truncation the expansion (9) in order to obtain an approximate solution.

2. 3 MAIN RESULTS AND DISCUSSION. Nutation amplitudes for different maximum numbers of characteristic functions in (9) are given in table 1 . For comparison, nutation amplitudes for the rigid and Poincare models are also shoun.

Table 3. Nutation amplitudes $n$ in angular milliseconds $\left(\delta=0.2, \varepsilon_{c}=0.0715\right)$ for an Earth with rigid mantle.

\begin{tabular}{|c|c|c|r|r|r||}
\hline \multirow{2}{*}{$\omega$} & \multirow{2}{*}{$\begin{array}{c}\text { Solid } \\
\text { model }\end{array}$} & $\begin{array}{c}\text { Poincare } \\
\text { model }\end{array}$ & \multicolumn{3}{|c|}{ Compressible core model } \\
\cline { 3 - 6 } & & & $M=2$ & $M=4$ & $M=6$ \\
\hline-6800 & 8051.05 & 7999.60 & 8000.851 & 8000.848 & 8000.848 \\
-365.3 & 24.94 & -38.633 & -25.536 & -25.552 & -25.554 \\
-182.6 & 22.60 & 28.197 & 28.329 & 28.329 & 28.329 \\
\hline
\end{tabular}

The results obtained show that the compressibility of the fluid Earth core may significantly affect theoretical nutation amplitudes and must be taken into account while calculating nutation amplitudes. Comparison of theoretical nutation amplitude with radiointerferometer data may give some information about the Earth core. 
3 NUTATION OF EARTH WITH SOLID INNER AND FLUID OUTER CORES

3. 1 FORMULATION OF THE PROBLEM. Let us investigate a symplified earth model consisting of: (i) rigid mantle with ellipsoidal cavity; (ii) ideal homogeneous incompressible liquid, filling the cavity; (iii) solid inner core, which is under the influence of the gravitation field in the center of the cavity.

Let the surface of the cavity and the surface of the inner core be discribed by the equations:

$x^{2}+y^{2}+z^{2} /\left(1-\varepsilon_{1}^{2}\right)=R_{1}^{2}$.

$x^{2}+y^{2}+z^{2} /\left(1-\varepsilon_{2}^{2}\right)=R_{2}^{2}$.

Here $R_{1}$ and $R_{2}, \varepsilon_{1}$ and $\varepsilon_{2}$ are equatorial radiuses and eccentricities of cavity and solid core correspondingly.

Let us examine the system behavior under the action of the tide-generating potential. The angular velocity of mantle rotation is of the form:

$\Omega=\{\eta(1-i j) \operatorname{ext}(i \sigma t)+k\} \omega$,

Motion of the inner core with respect to the moving coordinate system may be described by instantaneous angular velocity:

$\delta \omega=n_{1} \omega(1-i J) \exp (i \sigma t)$

Unknown amplitudes of nutation $n$ and $n_{1}$ must be determined from the solution of the problem.

The small oscillations of the fluid core are described by the following equation:

$\Delta \psi-\frac{4 \omega^{2}}{\sigma^{2}} \frac{\partial^{2} \psi}{\partial z^{2}}=0$,

with boundary conditions of the form:

$$
\begin{gathered}
\hat{\mathbb{B}}_{\mathrm{o}} \psi=\frac{i k \sigma n}{2\left(1-k^{2}\right) R_{1}}\left(1-\frac{1}{k}-\frac{\tau^{2}}{1-\varepsilon_{1}^{2}}\right)(x z-i y z), \quad r \in \mathrm{S}_{1} \\
\hat{\mathbb{B}}_{\mathrm{a}} \psi=\left[\frac{i k \sigma n}{2\left(1-k^{2}\right) R_{2}}\left[1-\frac{1}{k}-\frac{\tau^{2}}{1-\varepsilon_{2}^{2}}\right]+\frac{i \eta_{1} \omega}{R_{z}} \frac{\varepsilon_{2}^{2}}{1-\varepsilon_{2}^{2}}\right](x z-i y z), r \in \mathrm{S}_{2} \\
\hat{\mathbb{B}}_{\mathrm{O}} \psi=\frac{-i k}{2 \omega\left(1-k^{2}\right) R}\left\{\left[x-\frac{y}{i k}\right) \frac{\partial \psi}{\partial x}+\left[v+\frac{x}{i k}\right) \frac{\partial \psi}{\partial y}+\frac{\tau^{2} z}{1-\varepsilon^{2}} \frac{\partial \psi}{\partial z}\right\} \\
k=\sigma / 2 \omega, \quad \tau=1-1 / k^{2} .
\end{gathered}
$$

Here $S_{1}$ and $S_{2}$ are the surfaces of the cavity and the solid core correspondingly. 
3.2 RESULTS. Approximate solution of the boundary value problem (7), (9) may be written in the form:

$\psi \approx\left(a_{1}+a_{2}\right)(x z-i y z)$.

$a_{1}=-$ now $\frac{1-1 / k-\tau^{2} /\left(1-\varepsilon_{1}^{2}\right)}{1-1 / k+\tau^{2} /\left(1-\varepsilon_{1}^{2}\right)}$

$a_{2}=-\frac{\text { now }}{h_{2}^{*}}\left\{\left(1-\frac{1}{k}-\frac{\tau^{2}}{1-\varepsilon_{2}^{2}}\right)-\left(1-\frac{1}{k}+\frac{\tau^{2}}{1-\varepsilon_{2}^{2}}\right)_{1-1 / k+\tau^{2} /\left(1-\varepsilon_{1}^{2}\right)}^{1-1 / k-\tau^{2} /\left(1-\varepsilon_{1}^{2}\right)}\right\}-$

$$
-\frac{2 \eta_{1} \omega^{2}\left(1-k^{2}\right)}{k h_{2}^{*}} \frac{\varepsilon_{2}^{2}}{1-\varepsilon_{2}^{2}}
$$

$-h_{2}^{*} R_{2}^{5}=\left\{\left(1-\frac{1}{k}+\frac{\tau^{2}}{1-\varepsilon_{1}^{2}}\right) R_{1}^{5}-\left[1-\frac{1}{k}+\frac{\tau^{2}}{1-\varepsilon_{2}^{2}}\right) R_{2}^{5}\right\}$

In table 2 there are represented the values of nutation amplitudes for: (i) the solid Earth model, (ii) the Poincare model, (iii) the examined model. Numbers in Table 2 shows that correction to forced nutation amplitude due to the existance of the inner core, may be greater than the accuracy of observations.

Table 1. Nutation amplitude in angular miliseconds $\left(r_{2}=0.357 r_{1}, \varepsilon_{1}=0.0715, \varepsilon_{2}=0\right)$.

\begin{tabular}{|c|c|c|c|}
\hline$\frac{\omega}{\sigma+\omega}$ & $\begin{array}{c}\text { Rigid } \\
\text { model }\end{array}$ & $\begin{array}{c}\text { Poincare } \\
\text { model }\end{array}$ & $\begin{array}{c}\text { Solid inner } \\
\text { core model }\end{array}$ \\
\hline-6800 & 8051.05 & 7999.92 & 8000.23 \\
-365.3 & 24.91 & -38.63 & -31.24 \\
-182.6 & 22.60 & 28.16 & 28.20 \\
182.6 & 530.80 & 571.71 & 571.63 \\
\hline
\end{tabular}

\section{REFFERENSES}

[1] Smith M.L., 1974. The scalar equations of infinjtesimal elastic-gravitational motion for a rotating slightly e1liptical Earth., Geoph. J. R. astr. Sos., 37, 491-526.

[2] Crossly D.J., Rochester M.G., 1980, Simple core undertones., Geoph. J. R. astr. Sos., $60,129-161$.

[3] Wahr J.M.,1981, The forced nutation of an elliptical, rotating elastic and oceanless Earth., Geoph. J. R. astr. Sos., 64, 705-727.

[4] Bykova V.V..1990, Proceedings of the IAU Colloguium 127. in press. 